\title{
LA TRADUCCIÓN A LA VISTA EN EL ÁMBITO MÉDICO-SANITARIO: UN CASO DIDÁCTICO
}

\author{
Gianluca Pontrandolfo \\ gpontrandolfo@units.it \\ Universidad de Trieste - IUSLIT
}

\section{Resumen}

El presente estudio examina un caso didáctico que recrea fielmente una mediación profesional auténtica llevada a cabo en un hospital de Trieste. El análisis se basa en una muestra de transcripciones de traducción a la vista (TAV) realizadas por estudiantes del último curso de la Laurea Magistrale en Traduzione Specialistica e Interpretazione di Conferenza (curriculum integrato) de la Universidad de Trieste. El foco del análisis recae en dos géneros que el mediador sanitario tiene que manejar con cierta soltura: a) el formulario de consentimiento informado [TAV it>es cuyo destinatario es el paciente]; b) la historia clínica [TAV es>it cuyo destinatario es el personal médico]. A partir de una reflexión sobre el producto de traducción (las reformulaciones realizadas por el alumnado), en términos de problemas, errores y estrategias, el análisis detecta las áreas más problemáticas de la TAV y brinda consideraciones preliminares para la formación de mediadores lingüísticos y culturales.

\begin{abstract}
"Sight Translation in healthcare settings: a case-study"

This paper focuses on a training case-study which simulates a professional mediation carried out in a hospital in Trieste. The analysis is based on a collection of transcripts of sight translations performed by students in their last year of the MA in Specialised Translation and Conference Interpreting (mixed curriculum) of the University of Trieste. The focus of the analysis is on two genres which the mediator has to manage with ease: a) the informed consent form [sight translation it>es whose addressee is the patient]; b) the clinical history [sight translation es>it whose addressee is the medical staff]. By reflecting upon the translation product (i.e. the students' renditions) in terms of problems, errors and strategies, the paper identifies the most problematic areas and provides preliminary considerations for the training of linguistic and cultural mediators and interpreters.
\end{abstract}


Palabras clave: Traducción a la vista (TAV) en el ámbito sanitario. Simulación didáctica. Problemas. Errores. Estrategias.

Keywords: Sight translation in healthcare setting. Training simulation. Problems. Errors. Strategies.

Manuscript received on April 11, 2015 and accepted for publication on September 19, 2015. 


\section{Premisas}

Una de las tareas que más a menudo tienen que llevar a cabo los mediadores lingüísticos y culturales ${ }^{1}$ en el ámbito médico-sanitario es la traducción a la vista (TAV) (véase, entre otros, Jiménez Ivars \& Hurtado Albir 2003) de documentos esenciales para la realización de distintas actividades, desde una simple consulta médica hasta una delicada intervención en el quirófano.

En la mayoría de los casos, al mediador no se le proporciona el texto de antemano y eso le obliga a traducir a la vez que lee por primera vez (traducción a ojo o a primera vista, Jiménez Ivars \& Hurtado Albir 2003: 50), actividad que genera una serie de dificultades y requiere la adopción de distintas estrategias.

Aunque representa una de las modalidades traductoras más empleadas en contextos sanitarios profesionales, la atención de la comunidad investigadora no se ha centrado mucho en la TAV ni en traductología (Hurtado Albir 2011: 83), ni en los estudios sobre la interpretación/mediación en el ámbito médico (Niemants 2012; Valero-Garcés et al. 2014), y además, ni desde la perspectiva didáctica (véanse, entre otros, Zorzi 2007; Carreras i Goicoechea \& Pérez Vázquez 2009) ni desde la vertiente profesional (véanse, entre otros, Valero Garcés 2008; Burckhardt et al. 2009; Rodríguez Cala \& Llevot Calvet 2011; Iacono 2013). Desde la vertiente académica, la TAV sigue representando una estrategia pedagógica (Jiménez Ivars \& Hurtado Albir 2003: 54), es decir, un instrumento más que una modalidad traductora y, por tanto, una actividad propedéutica de la interpretación simultánea y/o consecutiva (véanse, entre otros, Viezzi 1989; Weber 1990; Moser-Mercer 1994; Viaggio 1995; Ballardini 1998). Por lo que se refiere al ámbito médico-sanitario, no existen, hasta hoy, investigaciones sistemáticas que aborden el delicado tema de la TAV en dicho sector.

El presente estudio sienta sus bases en una simulación didáctica que recrea fielmente una mediación profesional real. El análisis se basa en una

1. Para Pöchhacker (2008: 11 y ss.) la mediación lingüística es inevitablemente mediación cultural. 
muestra de transcripciones de TAV realizadas por estudiantes del último curso de Máster (Laurea Magistrale en Traduzione Specialistica e Interpretazione di Conferenza- curriculum integrato) de la Universidad de Trieste. El foco del análisis se dirige a dos géneros que el mediador sanitario tiene que manejar con cierta soltura:

a) el formulario de consentimiento informado (Cecilia Ramos 2012);

b) la historia clínica (De la Prieta Miralles 2002).

En a) el destinatario de la TAV es el paciente, mientras que en b) es el personal médico-sanitario; este cambio de función del texto y destinatario -lego vs. experto- tendría que afectar, evidentemente, a las estrategias y técnicas empleadas por el mediador.

A partir de una reflexión sobre el producto de traducción (las reformulaciones realizadas por el alumnado), en términos de problemas, errores y estrategias (Jiménez Ivars 1999), el análisis detecta las áreas más problemáticas de la TAV y brinda consideraciones preliminares para la formación de mediadores lingüísticos y culturales (Isasa 2014).

En el marco de la mediación entre lenguas afines, español-italiano (Bazzocchi \& Capanaga 2006), el estudio combina los dos enfoques a la TAV: profesional (TAV con función comunicativa) y pedagógico (TAV con función instrumental) (Jiménez Ivars \& Hurtado Albir 2003: 47-49).

En los apartados siguientes, tras presentar los cimientos teóricos y metodológicos de la investigación realizada (§ 2), se esboza el diseño del estudio, haciendo hincapié en el perfil del alumnado, la tipología de encargo, el experimento de TAV y el cuestionario ( $\S 3$ ). En $\S 4$ se presentan los resultados, desglosados mediante un análisis cuantitativo y cualitativo, mientras que en $\S 5$ se hace un rápido balance del estudio y se brindan consideraciones finales y propuestas para investigaciones futuras.

\section{Los cimientos: preguntas de investigación, hipótesis y objetivos}

Las preguntas de investigación que han inspirado el estudio se enmarcan tanto en el ámbito profesional como didáctico. Con respecto al primero, sería interesante abordar las siguientes preguntas preliminares:

1) ¿Cuáles son las características de esta modalidad traductora (con respecto, por ejemplo a la interpretación bilateral)?

2) ¿Qué tipologías de problemas engendra y cuáles son las estrategias de gestión típicas del ámbito médico-sanitario? 
Desde el punto de vista didáctico, y en estricta conexión con las preguntas precedentes, la pregunta inspiradora es la siguiente: ¿Están listos los recién licenciados de Trieste para trabajar como mediadores en el ámbito médico-sanitario?

A este respecto, se parte de la hipótesis de que los alumnos no están listos para gestionar encargos de TAV en el ámbito médico-sanitario y necesitan una formación ad hoc: la actividad es altamente compleja no solamente por las dificultades técnicas del ámbito médico-sanitario - p. ej., la terminología, que, como se deduce de $\S 4.3$, representa la preocupación principal y la principal fuente de estrés para el alumnado-, sino también porque, más sencillamente, los aprendices no están acostumbrados a esta modalidad traductora y apenas tienen experiencia didáctica en este campo (véase § 4.3).

La relevancia y justificación del estudio radica en la necesitad de mapear el campo desde una triple perspectiva: por una parte, es fundamental conocer las necesidades de los usuarios (tanto el personal sanitario como los pacientes); por otra, desde la perspectiva del alumnado, es fundamental que los aprendices sepan reconocer sus áreas débiles para potenciarlas, si se quiere trabajar en este ámbito profesional; por último, desde el punto de vista del profesorado, puede resultar útil reconocer cuáles son las exigencias formativas específicas de los alumnos para que en consecuencia puedan organizar sus clases.

Con respecto al objetivo, cabe distinguir entre uno a corto plazo, es decir, brindar propuestas específicas a partir de las exigencias formativas y las áreas débiles del alumnado, identificadas en el marco del estudio, y otro a largo plazo, o sea, mejorar la calidad del servicio de mediación proporcionado y formar de manera más puntual a los mediadores intentando establecer puentes de comunicación entre el mundo médico-sanitario y el mundo académico (formación de mediadores).

\section{El estudio}

\subsection{El perfil del alumnado}

Con el objetivo de identificar problemas, errores y estrategias en una simulación de encargo real de TAV se ha llevado a cabo un experimento que ha contado con la participación de una muestra reducida de alumnos. ${ }^{2}$ Se trata,

2. Se comparte la afirmación de Gile (1991: 60), citado en Jiménez Ivars (1999: 240): "Students can be used when enough evidence is available to indicate that they are comparable to professionals in the type of behaviour under study". 
más específicamente, de 8 alumnos de la Laurea Magistrale en Traduzione Specialistica e Interpretazione di Conferenza (curriculum integrato) a punto de licenciarse que han cursado la asignatura Traducción especializada español-italiano II. El módulo de 30 horas se centra en la traducción médica y saca partido de la diversificación "vertical" del discurso especializado (Calvi 2009: 28-32), según el enfoque de la aproximación gradual (Pontrandolfo, en prensa). Su objetivo es presentar las características de los distintos niveles de especialidad (Mapelli 2009: 113-120), desde la divulgación hasta los textos científicos, analizando los distintos géneros discursivos, desde el folleto divulgativo para pacientes (García Izquierdo 2009) hasta el artículo de investigación dirigido a expertos de medicina, pasando por los géneros intermedios como el consentimiento informado, la historia clínica, el prospecto de medicamentos, el caso clínico, etc. El grupo elegido para el experimento ya tiene conocimientos básicos del lenguaje médico (español e italiano) y ha traducido textos escritos complejos pertenecientes a este ámbito.

\subsection{El diseño del estudio}

El estudio se ha articulado en cuatro etapas:

a) Sesión informativa en la que a los alumnos se les explicaba el encargo ${ }^{3}$ de mediación (7 días antes de la simulación):

L'Unità Operativa Complessa di Chirurgia del Rachide "Sandro Agostini" dell'Azienda Ospedaliera Universitaria di Padova vi contatta per svolgere una mediazione linguistica durante un intervento chirurgico a cui dovrà sottoporsi una paziente boliviana. Il coordinatore infermieristico che vi contatta non vi fornisce molte informazioni sulla tipologia di intervento chirurgico. Vi riferisce soltanto quanto segue:

Si tratta di una giovane paziente boliviana con problemi di lombalgia. Le radiografie della colonna vertebrale sembrerebbero indicare una sacralizzazione di L5, alterazioni importanti di tipo degenerativo e, a livello L4-L5, la presenza di un'ernia discale. Sarà sottoposta tra una settimana a trattamento chirurgico di ernia discale lombare. Il vostro contributo sarà fondamentale non solo per la comunicazione medico/infermiere-paziente, ma anche nella ricostruzione della storia clinica della paziente.

La signora è arrivata da poco in Italia e non parla italiano; il personale medico e infermieristico non conosce lo spagnolo, ma riesce a cogliere qualche informazione per via dell'affinità tra le due lingue.

3. Sobre la importancia del translation brief en contextos profesionales reales, véanse Nord (1997a, 1997b: 46-48); Fraser (2000); Mossop (2007: 110-112). 
Aunque una semana para la preparación al encargo no es un plazo de tiempo real desde el punto de vista profesional, se ha creído suficiente y adecuado, considerando que se trata de un grupo de alumnos que empiezan a enfrentarse a contextos profesionales y no están acostumbrados todavía a trabajar a ritmos elevados típicos del mundo real. En esta misma línea, se coloca la decisión de proporcionar a los aprendices toda la información contenida en el encargo, si bien en contextos reales son pocos los mediadores que reciben todos esos detalles.

Es importante señalar que se ha decidido no desvelar la naturaleza del encargo (TAV) en esta etapa para evaluar en la fase final d) cómo se han preparado los alumnos y si se esperaban una TAV. Como se desprende del cuestionario (§ 4.3), 7 alumnos no habían pensado en esta modalidad traductora y esto tiene relevancia, mutatis mutandis, también para el ámbito médico-sanitario real, donde la TAV sigue empleándose mucho y donde los mediadores, muy a menudo, no se esperan traducir a ojo documentos médicos y, por tanto, no están preparados adecuadamente para el encargo.

b) Breve fase de introducción en la que se presenta al alumnado el contexto específico en el que va a desarrollarse la simulación (algunos minutos antes de la etapa c):

Sei arrivato/a in reparto.

Il coordinatore infermieristico ti spiega che il tuo contributo si articolerà in due parti:

1) TAV ES>IT: ricostruzione della storia clinica del paziente con l'infermiere specializzato e anestesista

2) TAV IT>ES: assistenza al paziente nella compilazione della "Dichiarazione di avvenuta informazione e consenso ad atto sanitario specifico", nello specifico, nell'atto relativo alle tecniche anestesiologiche.

In sostanza, dovrai informare il paziente circa il trattamento anestetico: esiti prevedibili dell'anestesia, rischi ed eventuali complicanze del trattamento e ulteriori informazioni di carattere anestesiologico (analgesia postoperatoria, problemi, procedure successive, etc.).

La fase b) representa una breve etapa de calentamiento, que responde a la necesidad de explicar, una vez más, el contexto en el que van a mediar y, sobre todo, aclarar que se trata de una traducción a la vista y no, como la mayoría se esperaba, de una simulación de una interpretación dialógica.

c) Simulación de la TAV propiamente dicha (es-it/it-es): cabe señalar que, en esta fase c), el alumnado no tenía posibilidad alguna de interactuar con el destinatario de la TAV y esto ha representado un reto adicional del encargo. 
d) Cuestionario final: el cuestionario final (§ 4.3) está parcialmente basado en Jiménez Ivars (1999: 350-351). Siguiendo a la autora, se ha concebido como un instrumento de medida de problemas detectados en la TAV, causas de los problemas y estrategias de resolución (1999: 242-243).

Los objetivos del cuestionario han sido dos: por una parte, evaluar la autopercepción del alumnado en relación con sus performances; por otra, identificar las dificultades subjetivas del alumnado (p. ej., la terminología) frente a las dificultades objetivas detectadas ex post (mediante un baremo de evaluación). Algunas preguntas del cuestionario estaban orientadas a definir el perfil del alumno (experiencia profesional, método de preparación, observaciones personales generales).

\subsection{Los textos}

Los textos elegidos para el experimento son textos reales producidos en el ámbito profesional.

\subsubsection{El texto español}

El primer texto (473 palabras) -elegido para la TAV español-italiano- recoge tres informes extraídos de la historia clínica de una paciente que acude al Hospital Clínico San Carlos de Madrid por problemas de lumbalgias: un informe radiológico del Servicio de Radiodiagnóstico del mismo hospital, un informe de urgencias y un informe de alta de urgencias. El destinatario de la $\mathrm{TAV}$, en este caso, es el personal médico-sanitario que necesita, a través del mediador, enterarse de la historia clínica de la paciente.

Para evitar dificultades adicionales a los alumnos, los textos originales de los informes que confluyen en la primera parte de TAV (es-it) han sido pasados al ordenador, ya que estaban escritos a mano por el personal médico del país de origen de la paciente.

Desde el punto de vista textual, los informes se presentan como textos medianamente complejos y exhiben muchas de las características del lenguaje médico (para el español, véanse, entre otros, Galán Rodríguez \& Montero Melchor 2002; Martín Camacho 2004; Vivanco Cervero 2006; Mapelli 2009; para el italiano: Serianni 2005, 2007: 89-106).

Por lo que se refiere al campo léxico-terminológico, destaca el uso de elementos léxicos condensados o abreviados (Mapelli 2009: 106): siglas (RX, L5, S1, 1N, N; siglas combinadas con símbolos: VHB +), acrónimos (U.P.A.) y abreviaciones (col. lumbar, anteced. pers., comp., Fdo., etc.), además de símbolos que corresponden a unidades de medidas ( $\mathrm{mg}, \mathrm{h}, 1 / 2$, etc.). 
Los tecnicismos puros se presentan como unidades simples (lumbalgia, cifoescoliosis, fisura, fractura; con sufijos tan productivos en el lenguaje médico como -ción: bipedestación, sedestación) y complejas (compuestos por disyunción -síndrome miccional, apófisis espinosas lumbares, ligamento peroneo astragalino, varo forzado, exploración general, resaltes óseos-, por sinapsia -sacralización de L5, fusión del arco posterior de S1-, contraposición-esguince-distensión- o yuxtaposición -columna lumbosacra-). Se señala también el empleo de denominaciones de medicamentos (generales: diclofármaco, ibuprofeno; y propias: diacepam, myolastan, Valium) y de dos términos marcados culturalmente (U.P.A., médico de atención primaria).

Desde el punto de vista morfosintáctico, cabe destacar el uso generalizado de la despersonalización, mediante el empleo de la $3^{a}$ persona, que difumina la figura del sujeto-locutor, de la pasiva refleja con se (se observa, se administró, se administra, se le explica, etc.) y de verbos con sujeto inanimado (Mapelli 2009: 107) (existir, presentar, apreciar, etc.).

Un rasgo típico del género textual "historia clínica" es la omisión de artículos, preposiciones, auxiliares que pueden deducirse del contexto y de los conocimientos compartidos por los interlocutores, sin menoscabo de la comprensión del texto (Mapelli 2009: 109), como se desprende de los siguientes ejemplos:

(1) Defecto de fusión del arco posterior de S1.

(2) Mujer de 31 años con anteced. pers. de lumbalgias ocasionales.

(3) No alergias medicamentosas conocidas.

(4) No síndrome miccional acompañante. Poco dolor en bipedestación.

(5) No dolor a la palpación sobre apófisis espinosas lumbares.

(6) No dolor en resaltes óseos.

La nominalización desempeña también un papel importante en el texto: sacralización de L5, alineación de cuerpos vertebrales, flexión de la columna lumbar, No dolor a la palpación, con dolor a la presión, etc., junto a la adjetivación explicativa (Mapelli 2009: 110): Radiología simple, partes blandas, Lumbalgia aguda, Mejoría clínica evidente, Poco dolor, lumbalgias ocasionales, adecuada alineación de cuerpos vertebrales, movilidad lateral, flexión ventral, etc.

Con respecto a los tiempos verbales, se observa una oscilación entre el presente de indicativo (se observa, existe, tiene, aporta, está, no puede, se administra, acude, no recuerda, etc.), que es el tiempo dominante ${ }^{4}$ en los

4. "El presente de indicativo [es] típico de las definiciones, las descripciones, la exposición y la observación de procedimientos y experimentos [...] [y] llega a dar un tono de universalidad y atemporalidad a los enunciados" (Mapelli 2009: 110). 
informes objeto de estudio, y el futuro (estar en combinación con el subjuntivo presente con idea de futuro: cuando esté; acudirá, etc.) y pretérito perfecto (no ha mejorado) e indefinido (se administró), con una escasa presencia de estructuras modales deónticas (Caminar con bastones de descarga, no apoyar pie al menos 1 semana).

Desde el punto de vista textual, los informes parecen poco cohesionados y coherentes y no manifiestan los rasgos que suelen exhibir los textos técnico-científicos, como la progresión temática lineal y constante del contenido (Mapelli 2009: 113). Además de la falta de coherencia en el uso de la $3^{\mathrm{a}}$ y $1^{\mathrm{a}}$ persona (p. ej., presenta aumento de partes blandas vs. no aprecio signos de fisura), cabe señalar el choque de registro que se produce en el documento redactado por el doctor. Al estilo impersonal y técnico se contrapone el estilo informal típico de la lengua hablada, como se desprende de los siguientes ejemplos:

(7) se ha agachado a meter unas cosas y ya no podía estirarse

caracterizado por el empleo de verba omnibus (Briz 1996: 44, 60), como el proverbo "meter" y del prosustantivo "cosas";

(8) La flexión ventral de la columna prácticamente no puede realizarla donde se evidencia el orden marcado de las palabras, que responde a la función pragmática de la topicalización y al realce informativo de los elementos (Briz 1996: 39). La dislocación a la izquierda está marcada por el clítico acusativo "la" y el tema que aparece en la periferia izquierda, típica de la lengua hablada (Briz 1998).

Si bien destaca la ausencia de marcadores discursivos y señalizadores, se señala la presencia de enumeraciones, útiles sobre todo en las secciones textuales que cada historia clínica dedica al tratamiento a seguir.

Se evidencian también faltas ortográficas (p. ej., en *zon referida, peroneo *astraglino, complementacion, etc.) que, junto a una puntuación casi ausente, dificultan la lectura del texto.

\subsubsection{El texto italiano}

El segundo texto (475 palabras) -escogido para la TAV italiano-español- es una sección de un consentimiento informado en uso en un hospital de Trieste ${ }^{5}$ que se refiere a la anestesia loco-regional (anestesia espinal y epidural), técnica

5. Su denominación oficial es: Dichiarazione di avvenuta informazione e consenso ad atto sanitario specifico; atto specifico: tecniche anestesiologiche. Se trata de un texto bastante estandarizado, como testimonian los modelos disponibles en la red. 
que, con toda probabilidad, se va a emplear en la intervención quirúrgica de la paciente simulada en el encargo. El destinatario de la TAV, en este caso, es el paciente que tiene que saber ${ }^{6}$ en qué consiste el tratamiento anestesiológico que le van a suministrar.

A diferencia del texto español, el italiano es mucho más estructurado, coherente y cohesionado. Su claridad estriba en su función o skopos (Nord 1997a): el destinatario final del texto es el paciente (no experto) que, antes de operarse, tiene que saber cómo funcionan las anestesias y cuáles son los beneficios y los problemas que de las mismas puedan derivar.

Desde el punto de vista terminológico, destaca la alternancia de tecnicismos de registro elevado (cefalea, anestesia generale inalatoria elo endovenosa, ematoma peridurale, etc.) y no elevado (riduzione della frequenza cardiaca en vez de bradicardia, calo della pressione arteriosa en vez de ipotensione, formicolio agli arti inferiori en vez de parestesia, etc.), con una prevalencia de estos últimos. La presencia de paréntesis explicativos en el texto confirma el corte típico de la comunicación médico-paciente (Montalt \& González Davies 2007: 230).

Muchos de los términos técnicos se refieren a sedes anatómicas (liquor cerebro-spinale, colonna vertebrale lombare, dura madre, osso sacro, colonna vertebrale toracica, etc.).

Por lo que atañe a los aspectos morfosintácticos, también en el texto italiano domina el estilo impersonal que intenta ocultar la presencia del agente (si ricorre ad una anestesia generale, è necessario passare all'anestesia, si associa a calo, viene spesso preceduta da, Liniezione viene praticata nello spazio peridurale, etc.). Con respecto a los tiempos verbales, domina el presente y el futuro en la parte final (il paziente sarà sorvegliato, sarà trasferito al reparto, etc.).

El texto se caracteriza por un alto porcentaje de estructuras modales con el verbo "poder", que expresan probabilidades, una estrategia de tutela empleada por el personal médico-sanitario en el caso de complicaciones que puedan surgir tras la anestesia (la complicanza più grave si può manifestare, Le anestesie peridurali possono causare aree di formicolio, etc.) y que llega a

6. La estructura sanitaria y los médicos están obligados por ley a proporcionar al paciente información adecuada y exhaustiva sobre el tratamiento médico (véanse el nuevo código de deontología médica de diciembre de 2006, capítulo IV, arts. 33/38; el Convenio Europeo sobre los derechos humanos y la biomedicina del Consejo de Europa del 4/04/1997, capítulo ii, arts. 5-6-8-15, y las recomendaciones de la Comisión bioética de la SIAARTI Società Italiana di Anestesia, Anelgesia, Rianimazione e Terapia Intensiva). 
su máxima expresión la oración que cierra el documento (Nessuna procedura anestesiologica può essere comunque priva di rischi).

Locuciones adverbiales como di regola y di norma (empleada dos veces) se insertan en la misma línea defensiva de autotutela contra el riesgo de complicaciones que puedan afectar a la salud de los pacientes.

Se señalan también estructuras condicionales que expresan hipótesis y probabilidades (Nel caso di un insufficiente effetto elo durata dell'anestesia regionale o di una sua eccessiva diffusione è necessario passare all'anestesia generale vera e propria. I Se invece sarà necessario un prolungamento del monitoraggio clinico e strumentale sarà trasferito al reparto di Terapia Intensiva secondo l'indicazione dell'anestesista-rianimatore).

Desde el punto de vista textual, la progresión temática es lineal: la atadura cohesiva es asegurada también por el empleo de marcadores discursivos (nel caso di, invece, pur, etc.), ausentes en el texto español, y por la recurrencia ${ }^{7}$ (la cui incidenza varia da 1,6 a 2 casi ogni 10.000 anestesie. Tale incidenza si è ridotta).

\subsection{Errores y estrategias}

La Tabla 1 recoge el baremo de evaluación empleado. La propuesta adapta e integra el baremo de Hurtado Albir (1999: 120), Mossop (2007: 125) y Hurtado Albir (2015). ${ }^{8}$

\begin{tabular}{|l|l|l|}
\hline (A) TRANSFERENCIA & (B) CONTENIDO & (C) LENGUAJE \\
\hline Exactitud [accuracy] & Lógica [logic] & Adecuación [tailoring] \\
Falso sentido (FS) & Contrasentido (CS) & Léxico (LEX) \\
No mismo sentido (NMS) & Sinsentido (SS) & Calcos (CAL) \\
Integridad [completeness] & Hechos [facts] & Sublenguaje [sub-language] \\
Adición (AD) & Errores factuales (FACT) & Terminología (TERM) \\
Supresión (SUP) & & Idiomaticidad [idiom] \\
& & Fraseología (FRAS) \\
& & Norma \\
& & Gramática (GRAM) \\
\hline
\end{tabular}

Tabla 1. Baremo de evaluación empleado para el análisis de los errores en la TAV

7. "La recurrencia es uno de los recursos cohesivos más empleados en los textos científicos y técnicos, ya que permite remarcar el tema del discurso y, por consiguiente, la coherencia del razonamiento" (Mapelli 2009: 111).

8. La terminología relativa al metalenguaje de la revisión está parcialmente basada en Parra Galiano (2005: 143). 
Por lo que atañe a las estrategias, se han adaptado las técnicas identificadas por Jiménez Ivars (1999: 252-255) al contexto médico-sanitario:

1. Omitir elementos de información

2. Seleccionar la idea fundamental

3. Evitar el mismo orden de palabras

4. Traducir palabra por palabra

5. Evitar el equivalente automático

6. Inventar

7. Avanzar en la lectura

8. Repetir, retomar el texto
9. Decir lo primero que viene a la mente

10. Releer la frase

11. Basarse en el contexto

12. Parafrasear

13. Generalizar

14. Razonar

15. Explicitar/Desterminologizar

\section{Análisis de los resultados}

El objetivo de esta sección es destacar los problemas de traducción más relevantes surgidos durante la simulación del encargo y las soluciones traductoras empleadas por el alumnado. Por razones de síntesis, se presentan los casos más llamativos, dejando el análisis puntual y pormenorizado para estudios futuros más extensos sobre el tema.

Por lo que atañe a la presentación de los resultados, se propone una sección dedicada a los errores ( $§ 4.1$ ), una a las estrategias ( $(4.2)$ y una al cuestionario (§ 4.3).

\subsection{Errores}

4.1.1. Errores en la TAV ES>IT: análisis cuantitativo y cualitativo

El análisis cuantitativo de los errores cometidos por los estudiantes en la dirección español-italiano evidencia las áreas más problemáticas (véase la Figura $1)$ : omisiones (30\%), falsos sentidos (21\%) y calcos (18\%). Hay que distinguir entre la omisión voluntaria de información -estrategia (véase § 4.2) - y la omisión debida a una dificultad de comprensión del texto origen (TO). En este segundo caso, se trata de las omisiones debidas a la comprensión del texto que Barik (1994) denomina comprehension omissions ${ }^{9}$ y que generan una pérdida de significado importante.

9. Barik distingue entre las siguientes categorías de omisiones detectadas en la interpretación simultánea: skipping omissions (supresión de una palabra, muy a menudo un adjetivo calificativo o de un enunciado que no altera la estructura gramatical ni determina pérdidas de significado); comprehension omissions (supresiones debidas a la falta 
Es interesante notar que la terminología (10\%) no representa un escollo significativo, en claro contraste con las dificultades percibidas por el alumnado (véase § 4.3).

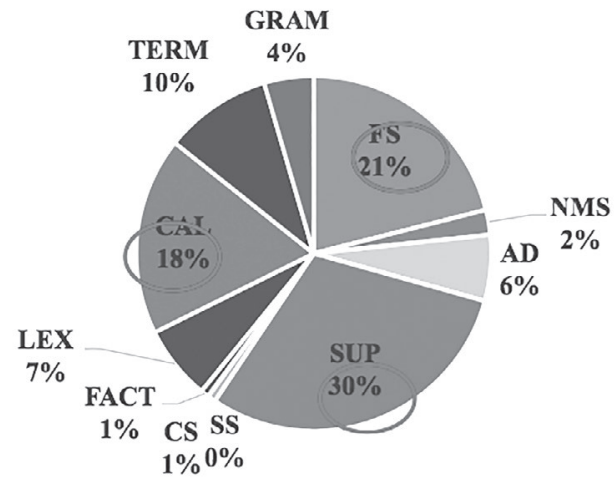

Figura 1. Errores en la TAV ES>IT

A continuación se presentan ejemplos relacionados con las tipologías de errores más frecuentes (omisiones, falsos sentidos y calcos).

(9) Exploración general: Presenta aumento de partes blandas en zona referida con dolor a la presión y en la flexión plantar y varo forzado

T1: la visita generale mostra un aumento di (.) di di parti (.) blande eh (.) eh riferisce dolore alla pressione e alla flessione plantare eh: (.) [SUP]
(10)

Enfermedad actual:

Acude por dolor en antepie [sic], más a nivel de ligamento peroneo astrag $[a \mid$ lino, no recuerda antecedente traumático.
T6: eh: si è recata al eh: pronto soccorso perché registr- perché soffriva di eh: un dolore ehm: in posizione ehm: (.) (DEGLUTISCE) soffriva di un dolore della colonna vertebrale [ $\underline{F S}$ ] (.) eh: (.) più al livello del: legamento peroneo as- del legamento peroneo [SUP] e non si ricorda di nessun precedente traumatico.

de comprensión por parte del intérprete y que determinan una interrupción y pérdida semántica); delay omissions (supresiones debidas al retraso de traducción del texto por parte del intérprete que, para seguir el ritmo del texto original, omite algunas de sus partes); compounding omission (el intérprete combina elementos pertenecientes a dos enunciados diferentes, omitiendo partes de ellos; el contenido resulta ser parcialmente alterado). 
En el ejemplo (9) la omisión [SUP] es debida a un problema terminológico: el alumno Tl no conoce la maniobra del varo forzado (en italiano 'manovra in varo equino forzato') que afecta la rodilla y que se utiliza para descartar lesiones del ligamento lateral externo y omite que la paciente refiere dolor también durante dicha maniobra.

En el caso del ejemplo (10) la omisión del adjetivo "astragalino" (en el texto original falta la [a]) determina una generalización semántica, ya que no se especifica la sede puntual del dolor en el ligamento peroneo. Se señala también el falso sentido producido por la traducción de "dolor en antepié" con "dolore della colonna vertebrale" lo cual confirma que el alumno T6 no tiene clara la anatomía del cuerpo humano, aspecto fundamental que cada mediador que trabaje en el ámbito médico-sanitario no puede no conocer.

Informe de alta de urgencias Hora llegada: 14.03

Hora de alta: 14.42

T3: dunque per quanto riguarda eh la: il ricovero eh la paziente è ararrivata alle quattordici e tre ed è stata ricoverata $[\mathrm{FS} / \mathrm{CS}]$ alle quattordici e quarantadue

(12)

\section{Datos clínicos:}

Dolor lumbar

RX COL. LUMBAR

Informe:

Como variante anatómica $[\ldots]$
T5: La paziente accusava dolore nella zona: lombare, eh: in particolare nella parte destra [FS][SUP] ed è stato eh: rilevato-a una sacralizzazione di L5.

(13)

Tratamiento al alta:

- Hielo local según se le explica

T1: il trattamento dopo: la lu- l'uscita eh: ghiaccio (.) ghiaccio s- (.) la nella zona affetta quand- a quando ce n'è bisogno [FS]

Los ejemplos (11), (12) y (13) presentan errores graves [FS] ya que el oyente (en el caso simulado, el personal sanitario) no puede darse cuenta de la inexactitud semántica. En (11), el horario de alta se interpreta como horario de hospitalización y no como horario de salida del hospital; en (12) "RX" unánimemente conocida como sigla para indicar la radiografía es interpretada, en cambio, como "parte derecha" (aunque en español su abreviatura sería 'dcha.'); en (13) la modalidad de aplicación del hielo según las indicaciones del personal médico cambia en la versión de Tl, que la interpreta de manera distinta: hielo en el caso de que haga falta.

Los problemas de interferencia y, en particular, la tendencia al calco léxico, morfosintáctico o textual en el texto meta se presentan con una alta 
frecuencia por las afinidades entre español e italiano (Calvi 2001: 332). Los siguientes ejemplos presentan casos de calcos léxicos [CAL].

(14) INFORME:

Como variante anatómica se observa sacralización de L5. Existe una adecuada alineación de cuerpos vertebrales, sin pérdida de altura de cuerpos vertebrales ni de los espacios intervertebrales lumbares.
T4: come dati clinici viene riportato un dolore lombare e: il referto ah: appunto prevede una sacralizzazione della vertebra L cinque eh esiste: una: viene anche riportata un'adecuata(!) eh adeguata: allineazione [CAL] dei corpi vertebrali non c'è la la paziente non riporta perdita di: di altezza dei corpi vertebrali e nemmeno negli: negli spazi intervertebrali lombari

(15)

Se administró Diclofarmaco intramuscular a su llegada, que no ha mejorado la movilidad.

T1: si: le abbiamo amministrato [CAL] di- diclofarmaco intramuscolare al suo arrivo che però non non ha migliorato la mobilità

(16) Rx pie, no aprecio signos de fisura y/o fractura, lesiones calcificadas altura tarso astragalina.

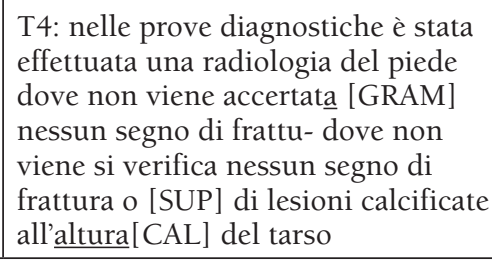
effettuata una radiologia del piede dove non viene accertata [GRAM] nessun segno di frattu- dove non viene si verifica nessun segno di frattura o [SUP] di lesioni calcificate all'altura [CAL] del tarso

Los ejemplos (14), (15) y (16) evidencian el influjo de la lengua origen en la lengua meta: "allineazione" en vez de 'allineamento', "amministrato" en vez de 'somministrato', "altura" en vez de 'altezza'.

Es interesante notar que en (14) el alumno T4 utiliza lo que Straniero Sergio (en prensa), en el marco de la interpretación simultánea, denomina double renditions (Dal Fovo 2013: 420-423), o sea, la producción de dobles traducciones equivalentes para expresar una misma unidad léxica del TO. En el corpus de análisis la mayoría de las ocurrencias de estos binomios está relacionada con la necesidad, por parte del alumnado, de acercarse al registro médico, come se desprende de (14) donde los verbos generales "esistere" y "essere" son remplazados enseguida por el verbo "riportare", típico del vocabulario médico. Otros casos detectados son ejemplos de autocorrección ("y permanecer en el cuarto donde sea donde está hospitalizado") o de aproximación al término más puntual y preciso ("Eh: además eh: hay eh: unos unos éxitos unos beneficios unos resultados del tratamiento"). 
Como destaca Straniero Sergio, muchas veces dichos binomios responden simplemente a una especie de autocomplacencia por parte de los intérpretes al demostrar dominio de la lengua y capacidad de "jugar con sinónimos" ante su ausencia de autonomía semántica. ${ }^{10}$

En (16) se señala también la falta de concordancia entre "accertata" y "segno" y la omisión de "fisura" en el doblete "fisura y/o fractura". La diferencia semántica entre los dos términos reside en que en la fisura la rotura del hueso no provoca discontinuidad entre los dos extremos (en italiano sería una 'lussazione'), mientras que en la fractura los dos extremos del hueso se separan (técnicamente una 'frattura' en italiano).

\subsubsection{Errores en la TAV IT>ES: análisis cuantitativo y cualitativo}

En la vertiente italiano-español, los errores más comunes se concentran en el campo lingüístico -calcos (46\%), errores gramaticales (25\%)- y semántico -omisiones (14\%) y falsos sentidos (8\%)- (Figura 2).

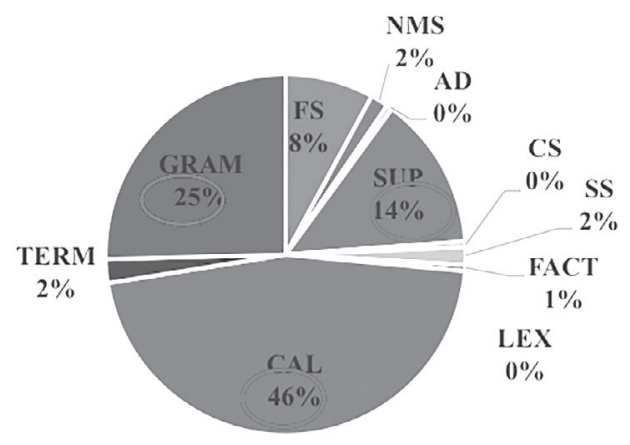

Figura 2. Errores en la TAV IT>ES

En la dirección italiano-español, casi la mitad de los errores se debe a interferencias lingüísticas. Los calcos [CAL] afectan no solamente los tecnicismos (17), sino también unidades léxicas de la lengua general (18).

10. "The practice of retroactive elaboration, whereby alternatives are continuously provided, may even reveal a sort of self-complacency on the part of the interpreter, in showing off his or her linguistic skills. 'Playing with synonyms', then, would compensate the interpreter's lack of semantic autonomy, foregrounding his or her role as a producer of a text of which s/he is not the owner" (Dal Fovo 2013: 423, citado por Straniero Sergio en prensa). 
$(17)^{11}$ reparto, anestesista, profesionista, cateterE, antidolorificos, anestesía, arteriosa (presión), suministrar, complicanzas, anestéTicos, terapÍa

(18) durada, monitoración, síngula (dosis), garantida, se efEctua, etc.

(19) Talvolta prima della procedura si ricorre ad una anestesia generale inalatoria e/o endovenosa, di norma senza intubazione.
T1: Eh: tal vez [CAL>FS] antes de la procedura [CAL] se recurre [CAL] eh: a una anestesia general eh: ehm: eh: (.) eh: que se eh: (.) eh. (:) que se aspira o: (.) en eh: (.) endovenosa.

En el ejemplo (19) el alumno T1 está completamente influenciado por su lengua materna y produce tres calcos consecutivos: "tal vez" (que genera también un falso sentido), "procedura" y "recurrir". ${ }^{11}$

Los problemas gramaticales, o sea, errores lingüísticos debidos a las diferencias estructurales entre español e italiano, representan otra categoría que se ha detectado con frecuencia en la TAV IT>ES.

Los ejemplos (20) y (21) evidencian errores gramaticales [GRAM] en el empleo del subjuntivo con valor de futuro (20) o de hipótesis (21).

(20)

Quando le sue condizioni cliniche saranno ritenute di assoluta sicurezza, potrà far ritorno al reparto di degenza.
T1: Cuando sus condiciones clínicas serán [GRAM] eh: seguras eh: podrá retorn podrá volver a: a su eh: reparto [CAL] [...].

(21)

Nessuna procedura anestesiologica può essere comunque priva di rischi, pur praticata da professionisti esperti, prudenti e attenti.
T4: Y: tenemos que decir que toda procedura [CAL] anestesiológica tiene algún riesgo eh: eh: aunque está [GRAM] practicada por profesionales expertos y eh: muy eh: atentos.

Con respecto a las supresiones [SUP] en la TAV IT $>$ ES, un caso interesante es el que proporciona el ejemplo (22). El texto en cuestión aparece en el TO al final y enmarcado en un cuadro con los caracteres en negrita, para subrayar que se trata de un elemento muy importante que el paciente debe conocer. Tres de ocho aprendices no tradujeron el texto, lo cual representa una omisión muy grave y que puede tener importantes consecuencias legales.

11. Se indican con letras mayúsculas las incorrecciones fonológicas de los alumnos, como por ejemplo anestesía o terapÍa con acento en vez de anestesia y terapia, o anestéTicos en vez de anestéSicos. 
(22)

\begin{tabular}{|l|l}
\hline Nessuna procedura anestesiologica & T3: [SUP]
\end{tabular} può essere comunque priva di rischi, $\quad$ T5: [SUP] pur praticata da professionisti esperti, $\quad$ T7: [SUP] prudenti e attenti.

(23) Liniezione viene praticata nello spazio peridurale (tra la dura madre e la parete del canale vertebrale) dove viene posizionato un sottile catetere a livello: $[\ldots]$

T6: la anestesia epidural eh: el fármaco se inyecta en el espacio peridural es decir entre eh: la: pared del canal vertebral y [SUP] el espacio: ehm: al lado [GEN] donde se posiciona un catéter muy sutil eh:

En (23) el alumno T6 omite la referencia al tecnicismo anatómico "dura madre" que se mantiene en la misma forma en español (duramadre).

\subsection{Estrategias}

4.2.1 Estrategias en la TAV ES>IT: análisis cuantitativo y cualitativo

Desde el punto de vista de las estrategias empleadas por el alumnado en la TAV de los informes al italiano, cabe destacar que la estrategia más empleada ha sido la generalización (31\%) seguida por la omisión (25\%) y la integración de información con finalidades fáticas (18\%).

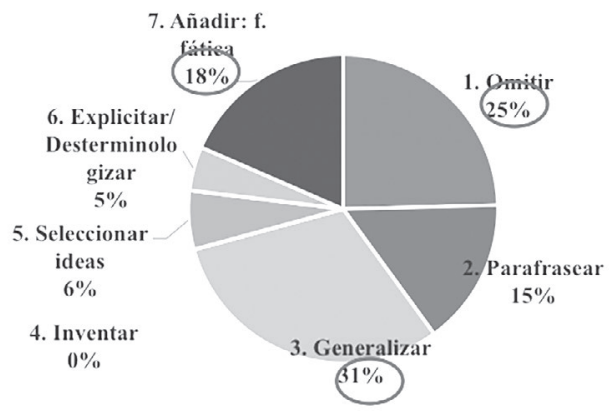

Figura 3. Estrategias en la TAV ES>IT

Por lo que se refiere a la dirección español-italiano, la estrategia de la generalización suele emplearse tanto para resolver problemas léxicos o terminológicos (24) como para acelerar la TAV y omitir información no esencial para la economía textual (25). 
(24) Tras la exploración, se administra

T2: Ehm: dopo la: dopo l'esame también 1 ampolla de Valium ${ }^{\circledR}$ fisico (DEGLUTISCE) eh: è stato (Diacepam). somministrato anche eh: un: mm: è stato somministrato anche un: [...] del [Generalización] valium (il diacepam)

(25) Código diagnóstico: Área: AREA 5 LA PAZ

Fecha ingreso: 29/07/2006 Hora: 19:38 Fecha alta: -

Especialidad: UNIDAD PRIMERA ASISTENCIA $1 \mathrm{~N}$

Parte judicial: Si/NO

Clave Hospital: 280066

T8: (.) a cui in seguito si trovano altri dati quali l'ora (.) e l'unità in cui è stata ricoverata (.) e quindi qui poi di seguito gli altri dati (.) [Generalización + Omisión]

En (24), el alumno T2 no sabe cómo traducir "ampolla" y generaliza utilizando el partitivo "del" (un poco de). Claramente, en contextos reales dicha estrategia no sería adecuada ya que la precisión de la información proporcionada al personal sanitario es fundamental para evaluar el tratamiento del paciente.

En (25), en cambio, el alumno T8 adopta la estrategia de la generalización para no detenerse en los datos que aparecen en el encabezamiento del informe, considerados no esenciales.

La estrategia de la generalización se relaciona con la estrategia de la paráfrasis, empleada con un porcentaje inferior (15\%) para resolver o evitar problemas terminológicos. La mayoría de los casos de paráfrasis detectados está asociada a dificultades de naturaleza terminológica, como se desprende de los ejemplos (26) y (27).

EXPLORACIÓN GENERAL:

Presenta aumento de partes blandas en zona referida con dolor a la presión y en la flexión plantar y varo forzado. No dolor en resaltes óseos.
T7: per quanto riguarda l'esame obiettivo ehm: si eh: (.) si dic- si descrive un: aumento di ehm: parti rilassate [TERM>FS] nella: nella zona eh interessata dal dolore ehm: per quanto riguarda la ehm: (.) la palpazione e la pressione effettuata dal personale medico [AD!] e una flessione plantare [SUP] ehm: ma non: non si riscontra dolore ehm: a livello: osseo [Paráfrasis] 
(27)

ENFERMEDAD ACTUAL:
Acude por dolor en antepie, más a nivel
de ligamento peroneo astraglino, no
recuerda antecedente traumático.

\author{
T2: Ehm: non ha mm: non ha problemi \\ particolari [Generalización] e per \\ quanto riguarda la sua malattia attuale \\ lei si presenta qui a visita per un dolore \\ $\mathrm{mm}:(. .$.$) nella parte: superiore del$ \\ piede [Paráfrasis + FS], più al livello del \\ legamento peroneo / più al livello del \\ legamento peroneo $[\ldots]$
}

En (26) el problema terminológico que determina la generalización es "resaltes óseos" que T7 traduce mediante el genérico "a nivel óseo". Además del error de supresión debido a la omisión de la maniobra del varo forzado, se señala también la adición no justificada de "presión efectuada por el personal médico", licencia que se toma el traductor sin saber si efectivamente el dolor a la presión era debido a una intervención del personal sanitario. Es más, dicha interpretación está en contra de las características del género discursivo (véase la despersonalización en § 3.3.1). Otro ejemplo de paráfrasis se encuentra en (27) para solucionar el problema terminológico "antepié", o sea, la parte anterior del pie, formada por los cinco metatarsianos y las falanges de los dedos correspondientes (equivalente del italiano "avampiede"). La elección del alumno es equivocada, ya que en la "parte superior" del pie no se suelen incluir los dedos.

Por lo que atañe a la segunda estrategia más empleada, la omisión, los casos se refieren casi siempre a elecciones conscientes de suprimir información considerada secundaria, como en el ya citado ejemplo (25), es decir, omisiones de encabezamientos de documentos en los que abundan siglas, códigos y números.

La estrategia de la adición fática es interesante porque confirma la intención del alumnado de mantener el control y el contacto del diálogo, a pesar de la situación poco real del encargo, donde no tenían posibilidades de interacción con el destinatario de la TAV (véase § 3.2). Si en (28) la adición no genera errores, dado que responde a la exigencia de introducir el documento, en (29), en cambio, las intervenciones autónomas del aprendiz no están justificadas y haber tomado la iniciativa genera problemas. ${ }^{12}$

12. En los estudios sobre la mediación en el ámbito médico-sanitario el tema del footing (el conjunto de posiciones y actitudes que el interlocutor puede asumir con respecto a los otros participantes en la interacción social, a lo que está ocurriendo y a sus propias palabras, Iacono 2014: 146) ya ha sido explorado a partir de los estudios de Goffman (1981: 325-326), Wadensjö (1998: 91-92), Merlini \& Favaron (2007: 116-117). Se remite a Iacono (2014) para un análisis claro y sintético de las principales alineaciones del intérprete-mediador en el ámbito médico-sanitario. 
(28)

\begin{tabular}{|l|l|}
\hline HOSPITAL CLINICO SAN CARLOS & T7: per quanto riguarda il secondo \\
INFORME DE URGENCIAS & $\begin{array}{l}\text { documento eh viene descritta in } \\
\text { maniera molto più eh dettagliata eh: } \\
\text { [... }\end{array}$ \\
& $\begin{array}{l}\text { quella che è la situazione eh della della } \\
\text { paziente ehm: (.) c'è una descrizione } \\
\text { ehm: }\end{array}$
\end{tabular}

(29)

23:45 - Mejoría clínica evidente

T7: alle ehm: alle undici e quarantacinque quindi eh parecchie ore dopo eh: [!] che la paziente è stata: si è rivolta al servizio di assistenza: sanitaria c'è eh c'è stato un miglioramento dal punto di vista clinico abbastanza evidente dovuto presumibilmente alla somministrazione del: farmaco [!]

En (29) una simple anotación se convierte en una explicación e interpretación arbitraria y autónoma del contexto: T7 no solo comenta el horario de mejoría del paciente ("muchas horas después del ingreso"), lo cual tiene una valoración subjetiva y, de alguna forma, negativa, sino que también comenta las razones de la mejoría, añadiendo que presumiblemente se trata de la acción del medicamento. Claramente, la estrategia adoptada en dicho contexto no es adecuada en absoluto, ya que el mediador no tiene que olvidar su papel y convertirse en doctor.

\subsubsection{Estrategias en la TAV IT>ES: análisis cuantitativo y cualitativo}

Con respecto a las estrategias adoptadas en la TAV al español, se señala una alta frecuencia de adiciones fáticas (27\%), aunque el resultado es, de alguna manera, influenciado por la elección de dicha estrategia por parte de un alumno en particular, lo cual altera los resultados globales. También la paráfrasis $(21 \%)$ y la generalización (18\%) se sitúan entre las estrategias más empleadas por parte de los aprendices, junto a la explicitación (16\%).

El empleo de dichas estrategias puede interpretarse como un esfuerzo, por parte de los alumnos, de tener en cuenta al destinatario de la TAV (el paciente), que es un lego y puede no conocer los tecnicismos de la materia. 


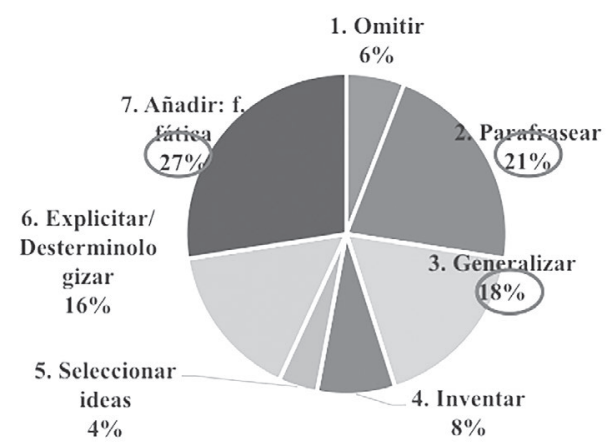

Figura 4. Estrategias en la TAV IT $>$ ES

Añadiduras fáticas son por ejemplo las que figuran en (30) y (31), donde T7 y T8 introducen lo que van a traducir para mantener un contacto con el destinatario (en este caso el paciente).

(30) 2. ANESTESIA LOCO REGIONALE $[\ldots]$

T7: bien eh: entonces aquí están algunos documentos que explican eh: lo que: eh: lo que es la: anestesia que: se va a realizar y: lo que son los efectos secundarios que puede que puede implicar.

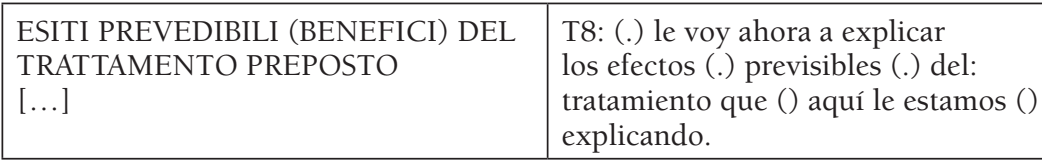

También en el caso de la TAV italiano-español, la estrategia consistente en parafrasear el contenido está relacionada con problemas léxico-terminológicos, como se desprende de los ejemplos (32) y (33). il paziente sarà sorvegliato per un periodo idoneo nella sala di risveglio.

T3: Después de la operación eh: el paciente será monitorizado durante un periodo idóneo en la sala en la que eh: se se despertara [Paráfrasis].

(33) Ulteriori informazioni di carattere anestesiologico. Analgesia postoperatoria $[\ldots]$
T1: Eh: más información de carácter eh: eh: de de de la anestesia eh: el eh: del tratamiento del dolor [Paráfrasis] postoperatorio 
En (32) la unidad léxica problemática es "sala di risveglio" (sala de recuperación), parafraseado por T3 con "en la sala en la que se despertara", donde el subjuntivo imperfecto resulta poco adecuado y transmite la idea de que la sala puede ser cualquiera y no una específica y dedicada a ese fin.

En (33) el término "analgesia" es parafraseado con "tratamiento del dolor" lo cual puede interpretarse bien como una paráfrasis debida a una dificultad terminológica, bien como estrategia consciente por parte del alumno, empleada para desterminologizar el término para el destinatario lego (Campos Andrés 2013). En efecto, la explicitación y la desterminologización han sido empleadas frecuentemente (16\%) por parte de los alumnos para facilitar la comprensión y recepción del texto por parte del paciente no experto (véase (34)).

\begin{tabular}{|c|c|}
\hline $\begin{array}{l}\text { Lanestesia spinale si associa a calo della } \\
\text { pressione arteriosa }(33 \%), \text { riduzione } \\
\text { della frequenza cardiaca }(13 \%) \text {, nausea } \\
(18 \%), \text { cefalea }(3,9 \%) .\end{array}$ & $\begin{array}{l}\text { T8: Los riesgos (.) del tratamiento } \\
\text { (.) son (.) puede bajar la la presión } \\
\text { (.) de la sangre (.) también (.) usted } \\
\text { podría sufrir de dolor de cabeza, de } \\
\text { mareo y también (.) podría bajar la (.) } \\
\text { frecuencia de su corazón }\end{array}$ \\
\hline
\end{tabular}

En el ejemplo (34) se nota cómo T8 explicita los tecnicismos médicos (arteriosa, frequenza cardiaca, cefalea) y elimina también los porcentajes, posiblemente por considerar que el destinatario lee a la vez el texto con el mediador y se da cuenta de la incidencia estadística medida en porcentajes de cada uno de los riesgos.

\subsection{Cuestionario: resultados}

El objetivo de este párrafo es presentar los resultados de las secciones más llamativas del cuestionario post-TAV rellenado por los aprendices.

Con respecto al método de preparación adoptado, la totalidad señala que la actividad principal para preparar el encargo fue la búsqueda en línea de textos especializados en ambas lenguas orientada a la elaboración de un glosario terminológico sobre el tema de la mediación. Como ya se ha señalado, la terminología representa, para el alumnado, el escollo principal de los textos médicos.

Es interesante notar que entre las expectativas del alumnado acerca de la tipología de mediación que se le iba a pedir no figuraba la TAV. De hecho, 7 de 8 alumnos no se esperaban una TAV, sino una clásica interpretación dialógica (con problemas de interacción paciente-personal sanitario), lo que confirma que la TAV sigue siendo una actividad infravalorada en contextos tanto didácticos como profesionales. 
En lo que atañe a la experiencia previa de encargos de TAV, desde la vertiente tanto didáctica como profesional, los datos proporcionados son relativamente bajos: una media de 12 horas en total, la mayoría de las cuales en las aulas universitarias como preparación a la interpretación bilateral y consecutiva. Se confirma la consideración inicial sobre la concepción de la TAV como actividad propedéutica más que modalidad traductora.

Otros parámetros interesantes son los datos relativos a la autoevaluación del nivel de comprensión del TO en relación con el nivel de dificultad percibido en la fase de producción de la TAV. La Tabla 2 sintetiza las puntuaciones medias de los alumnos.

\begin{tabular}{|c|c|c|c|}
\hline \multicolumn{2}{|c|}{ Nivel de comprensión del TO } & \multicolumn{2}{c|}{$\begin{array}{c}\text { Nivel de dificultad percibido en la } \\
\text { producción de la TAV }\end{array}$} \\
\hline ES & $7,2 / 10$ & ES $>$ IT & $7,2 / 10$ \\
\hline IT & $8,3 / 10$ & IT $>$ ES & $7,3 / 10$ \\
\hline
\end{tabular}

Tabla 2. Nivel de comprensión del TO y dificultad percibido por el alumnado.

Como se desprende de la Tabla 2, la comprensión del TO no representa un verdadero problema, mientras que el nivel de dificultad percibido en la fase de reformulación de los contenidos es alto. Para interpretar este dato cabe considerar las respuestas a las secciones siguientes del cuestionario, en las que se hace hincapié en los elementos de traducción problemáticos, sus causas y las soluciones empleadas para resolverlos.

Todos los problemas de traducción detectados pertenecen al área de la terminología: términos técnicos, siglas, acrónimos y unidades léxicas de uso frecuente en el lenguaje médico. Solamente 1 de los 8 alumnos indicó también problemas adicionales ligados a la dificultad para construir un discurso fluido debida a la modalidad TAV.

Con respecto a las causas es interesante notar que la causa principal explicitada en el cuestionario fue la comprensión de contenidos del TO (23\%), en neto contraste con los datos de la Tabla 2, la dificultad en encontrar el equivalente buscado (23\%) y la dificultad para mantener la debida distancia del TO en términos de interferencia (22\%).

En lo que atañe a la solución que los alumnos eligieron para resolver dichos problemas, la solución más mencionada fue la selección de la idea fundamental (30\%), seguida por otras soluciones que suponen el 34\% del total (p. ej, omisiones, proximidad al TO, empleo de sinónimos, retrasos en la traducción). 
Por último, respecto a la autoevaluación del grado de resolución de los problemas de traducción, en una escala de 0 (mal o no resuelto) a 3 (resuelto muy bien), la media de los resultados se sitúa en la parte baja: el $52 \%$ evaluó de manera totalmente negativa sus soluciones (0), el 37\% negativa (1) y el $11 \%$ positiva (2). Ninguno estuvo totalmente satisfecho con las soluciones adoptadas.

La Tabla 3 sintetiza la valoración global negativa de la TAV por parte de los aprendices, insatisfechos frente al encargo.

\begin{tabular}{|l|c|}
\hline \multicolumn{1}{|c|}{ Parámetros } & Autoevaluación \\
\hline exactitud (contenido) & $5,5 / 10$ \\
\hline integridad & $5,5 / 10$ \\
\hline expresión en IT & $6,1 / 10$ \\
\hline expresión en ES & $5,2 / 10$ \\
\hline sublenguaje (terminología) & $4,3 / 10$ \\
\hline idiomaticidad (fraseología) & $5,1 / 10$ \\
\hline percepción general & $4,7 / 10$ \\
\hline
\end{tabular}

Tabla 3. Autoevaluación del alumnado por parámetros.

Esta conciencia de las dificultades y de los errores cometidos representa un buen punto de partida para planificar programas de formación didáctica específica.

En la parte final del cuestionario, dedicada a observaciones generales sobre la actividad, todos los encuestados evidenciaron la "falta de naturalidad en la tarea", o sea, el hecho de que la situación simulada no era real, ya que, durante la TAV, no pudieron comunicar con el destinatario del texto y aclarar dudas.

Cabe subrayar que en la simulación realizada no se añadieron -voluntariamente- dificultades típicas de los contextos profesionales reales que hubieran complicado aún más el encargo, como, por ejemplo: la ya citada accesibilidad de los documentos, escritos a mano con la grafía del personal médico; problemas relacionados con el setting, o sea, el contexto espacial donde el mediador lleva a cabo sus tareas (en el quirófano, en la sala de espera, en el pasillo frente al quirófano, en la habitación del enfermo, etc.) y las prendas (batas, mascarillas faciales, etc.) que muchas veces tienen que llevar, los ruidos que suelen caracterizar dichos ambientes, etc.; dificultades relacionadas con las personas: la actitud del personal médico-sanitario y de los pacientes, que no siempre es previsible y que incide de manera significativa en la prestación del mediador; 
la rapidez con la que se desarrollan todos los procedimientos y las tareas; ${ }^{13} \mathrm{el}$ tiempo insuficiente de preparación para el encargo en comparación con los 7 días concedidos para preparar el experimento, un lapso temporal prácticamente irreal en la práctica profesional.

Si el alumnado ha tenido problemas en un contexto de relativa tranquilidad, en un lugar "aséptico", traduciendo sin ningún tipo de distracción y en total autonomía (con la única "molestia" del grabador de voz), es muy probable que, en contextos reales, no hubiera llevado a cabo el encargo de manera satisfactoria.

\section{Consideraciones conclusivas: un rápido balance}

Una evaluación holística del experimento debería considerar todos los aspectos relacionados con la calidad desde la vertiente profesional, un concepto polifacético que se puede enfocar desde distintos puntos de vista. Además de la correspondencia con el sentido del mensaje original, de la cohesión lógica de la versión, de la corrección en el uso terminológico y de la corrección gramatical -temas que han sido objeto de la presente contribución- habría que considerar también un elemento fundamental de toda interacción oral, es decir, el tema de la fluidez de la prestación. Es precisamente en esta área donde subyacen elementos cruciales como el tiempo, las pausas y las vocalizaciones, que han representado un obstáculo importante para el alumnado y que serán analizados con mayor profundidad en publicaciones futuras.

Volviendo a la pregunta inicial (¿Están listos los alumnos para trabajar como mediadores en el ámbito médico-sanitario?), el experimento realizado ha permitido confirmar la hipótesis inicial, o sea, que los aprendices no están acostumbrados a gestionar encargos de TAV y, por lo tanto, no están todavía listos para el mundo profesional sanitario.

Las áreas de mejora y, por ende, más relevantes para los profesores que trabajen en la combinación español-italiano, son las siguientes: interferencias (calcos de distinta tipología causados por afinidad entre los dos idiomas, pero también como estrategia de protección frente a los tecnicismos de la materia); accesibilidad (fruibilità) y naturalidad en la ejecución y producción del texto meta. En general, se revela necesaria una mayor conciencia del encargo y del papel fundamental desempeñado por el mediador.

13. Por razones de síntesis no se ha podido abordar el tiempo de la ejecución de la TAV que representa un factor crucial en toda mediación lingüística. Los aprendices tardaron una media de 11 minutos en cada encargo (ES>IT e IT>ES), un arco temporal demasiado largo para las exigencias médico-sanitarias. 
Ahora bien, hay que considerar también otro aspecto no secundario: no todos los mediadores que trabajan en el ámbito médico-sanitario tienen formación universitaria y preparación en el campo de la traducción y la interpretación. Los alumnos que hoy no están listos para trabajar profesionalmente podrán estarlo mañana, recibiendo una formación ad hoc en este sector, que haga hincapié no solamente en los aspectos lingüísticos y traductológicos, sino también en el lado psicológico de la profesión y en la gestión del estrés en contextos tan delicados como los sanitarios. Se trata de un auspicio para el futuro de la profesión; las recientes iniciativas en el ámbito académico italiano ${ }^{14}$ demuestran que el interés efectivamente está creciendo y que existe el potencial para atribuir a esta profesión, tan importante para una sociedad cada vez más multiétnica, el reconocimiento oficial que merece.

\section{Referencias bibliográficas}

BALLARDINI, Elio. (1998) "La traduzione a vista nella formazione degli interpreti." InTRAlinea 1. Versión electrónica: <http://www.intralinea.org/archive/article/ La_traduzione_a_vista_nella_formazione_degli_interpreti>

BARIK, Henri C. (1994) "A description of various types of omissions, additions and errors of translation encountered in simultaneous interpretation" En: Lambert, Sylvie \& Barbara Moser-Mercer (eds.) 1994. Bridging the gap: Empirical research in simultaneous interpretation. Ámsterdam \& Filadelfia: John Benjamins, pp. 121-137.

BAzzocCHI, Gloria \& Pilar Capanaga. (2006) Mediación lingüistica de lenguas afines: español/italiano. Bolonia: Clueb.

BRIZ, Antonio (2010 [1996]) El español coloquial: situación y uso. Madrid: Arco/ Libros.

BRIZ, Antonio. (1998) El español coloquial en la conversación. Barcelona: Ariel.

BurCKHARDT, Adil. Q; Hilda-Wara Revollo; Francisco Collazo; Cristina Wisiers Würth \& Jannat El Harrak. (2009) "La mediación intercultural sociosanitaria: implicaciones y retos." Norte de Salud Mental 35, pp. 56-66.

CALVI, Maria Vittoria. (2001) "La traduzione nell'insegnamento linguistico." En: Melloni, Alessandra; Rafael Lozano \& Pilar Capanaga (eds.) 2001. Interpretar, traducir textos de las culturas hispánicas. Bolonia: Clueb, pp. 327-342.

14. Un programa de formación interesante y específico es el Máster en Traducción y Mediación intercultural organizado por la Universidad de la Calabria (http://www.termcoord.eu/wpcontent/uploads/2014/11/traduzione-e-mediazione-interculturale.pdf) oelCursodeAlta Formación en Asistencia lingüística para elámbito judicial organizado por la Universidad de Bolonia (http://www.unibo.it/it/didattica/corsi-di-alta-formazione/2014-2015/ assistenza-linguistica-per-lambito-giudiziario-formazione-permanente). 
CAlvi, Maria Vittoria. (2009) "Las lenguas de especialidad." En: Calvi, Maria Vittoria; Cristina Bordonaba Zabalza; Giovanna Mapelli \& Javier Santos López (eds.) 2009. Las lenguas de especialidad en español. Roma: Carocci, pp. 15-38.

CAMPOS ANDRÉs, Olga. (2013) "Procedimientos de desterminologización: traducción y redacción de guías para pacientes.” Panace@ 14:37, pp. 49-52.

Carreras i Goicoechea, María \& María Enriqueta Pérez Vázquez (eds.) (2009) La mediación lingüística y cultural y su didáctica. Un nuevo reto para la Universidad. Bolonia: Bononia University Press. Versión electrónica: <http:// www.contrastiva.it/baul_contrastivo/dati/barbero/Iciar_Alonso_Baigorri.pdf>

CECILIA RAmos, Luciana. (2012) "El consentimiento informado."Panace@ 13:36, pp. 294-298. Versión electrónica: <http://www.medtrad.org/panacea/ IndiceGeneral/n36-revistilo_LCRamos.pdf>

DAL FOVO, Eugenia. (2013) "The language of interpreters on television: characteristics, tendencies, and idiosyncrasies.” En: Desoutter, Cécile; Dorothee Heller \& Michele Sala (eds.) 2013. Corpora in specialized communication Korpora in der Fachkommunikation Les corpus dans la communication spécialisée. Bérgamo: CELSB, pp. 411-434.

De la Prieta Miralles, Virtudes. (2002) "La historia clínica: aspectos lingüísticos y jurídicos.” Panace@ 3:8, pp. 36-39. Versión electrónica: <http://www. medtrad.org/panacea/IndiceGeneral/n8-PrietaMiralles.pdf>

FRASER, Janet. (2000) "The broader view: how freelance translators define translation competence.” En: Schäffner, Christina \& Beverly Joan Adab (eds.) 2000. Developing Translation Competence. Ámsterdam: John Benjamins, pp. 51-62

Galán Rodríguez, Carmen \& Jesús Montero Melchor. (2002) El discurso tecnocientífico: la caja de herramientas del lenguaje. Madrid: Arco/Libros

GARCÍA IZQUIERDO, Isabel. (2009) Divulgación médica y traducción: el género información para pacientes. Berna: Peter Lang.

GILE, Daniel. (1991) "Methodological Aspects of Interpretation (and Translation) Research." Target 3:2. pp. 153-174.

GOFFMAN, Erving. (1981) Forms of Talk. Oxford: Blackwell.

HurTAdo Albir, Amparo (ed.) (1999) Enseñar a traducir. Metodología en la formación de traductores e intérpretes. Madrid: Edelsa.

HurTado Albir, Amparo. (2011) Traducción y Traductología. Introducción a la traductología. Quinta edición revisada. Madrid: Cátedra.

Hurtado AlBiR, Amparo. (2015) Aprender a traducir del francés al español. Competencias y tareas para la iniciación a la traducción. Madrid \& Castellón: Edelsa \& Univ. Jaume I.

IACONO, Eleonora. (2013) Interazioni, ruoli e strategie di cortesia nell'interpretazione italiano-spagnolo in campo medico. Palermo: Università degli Studi di Palermo. Tesis doctoral. Versión electrónica: <https://iris.unipa.it/retrieve/ 
handle/10447/102412/137469/Tesi\%20dottorale\%20Iacono\%20Eleonora. pdf>

IACONO, Eleonora. (2014) "La alineación verbal del intérprete en un corpus de entrevistas médicas español-italiano." Estudios de Traducción 4, pp. 145160. Versión electrónica: <http://revistas.ucm.es/index.php/ESTR/article/ viewFile/45373/42693>

ISASA, Luna. (2014) "Formación del profesional sanitario en la comunicación con el paciente extranjero: estrategias y recursos.” Panace@ 15:40, pp. 243257. Versión electrónica: <http://www.medtrad.org/panacea/IndiceGeneral/ n40_tribuna_Isasa.pdf >

JimÉnEZ IVARS, Amparo. (1999) La traducción a la vista. Un análisis descriptivo. Castellò de la Plana: Universitat Jaume I. Tesis doctoral. Versión electrónica: <http://repositori.uji.es/xmlui/bitstream/handle/10234/29703/jimenez-tdx. pdf? sequence $=1>$

JimÉNEZ IVARS, Amparo \& Amparo Hurtado Albir. (2003) "Variedades de traducción a la vista. Definición y clasificación." Trans 7, pp. 47-57. Versión electrónica: <http://www.trans.uma.es/Trans_7/t7_47-57_AJimenez.pdf>

MAPELli, Giovanna. (2009) "El lenguaje técnico-científico." En: Calvi, Maria Vittoria; Cristina Bordonaba Zabalza; Giovanna Mapelli \& Javier Santos López (eds.) 2009. Las lenguas de especialidad en español. Roma: Carrocci, pp. 101-120.

MARTín CAMACHO, José Carlos. (2004) El vocabulario del discurso tecnocientífico. Madrid: Arco/Libros.

MERLini, Raffaella \& Roberta Favaron. (2007) "Examining the 'voice of interpreting' in speech pathology." En: Pöchhacker, Franz \& Miriam Schlesinger (eds.) 2007. Healthcare Interpreting. Ámsterdam \& Filadelfia: John Benjamins, pp. 101-137.

Montalt, Vincent \& María González Davies. (2007) Medical translation step by step: learning by drafting. Manchester: St. Jerome.

Moser-MerCer, Barbara. (1994) "Aptitude testing for conference interpreting: Why, when and how." En: Lambert, Sylvie \& Moser-Mercer, Barbara (eds.) 1994. Bridging the gap: Empirical research in simultaneous interpretation. Ámsterdam \& Filadelfia: John Benjamins, pp. 57-68.

Mossor, Brian. (2007) Revising and Editing for Translators. Manchester: St. Jerome. NiEMANTS, Natacha. (2012) Traduzione e mediazione nell'interpretazione dialogica in ambito sanitario: ruolo o responsabilità? Una risposta interazionista. Modena: Università degli Studi di Modena e Reggio Emilia. Tesis doctoral. Versión electrónica: <http://www.dailynterpreter.com/phd-thesis.pdf >

NORD, Christiane. (1997a) Translation as a Purposeful Activity. Functionalist Approaches Explained. Manchester: St. Jerome. 
NORD, Christiane. (1997b) "Defining Translation Functions. The Translation Brief as a Guideline for the Trainee Translator." En: Lörscher, Wolfgang (ed.) 1997. Translation Studies in Germany. Ilha do Destierro, Special Issue, pp. 39-53. Versión electrónica: <https://periodicos.ufsc.br/index.php/desterro/ article/download/9208/9484>

PARRA GALIANO, Silvia. (2005) La revisión de traducciones en traductología: aproximación a la práctica de la revisión en el ámbito profesional mediante el estudio de casos y propuestas de investigación. Granada: Universidad de Granada. Tesis doctoral. Versión electrónica: <http://hera.ugr.es/tesisugr/15472905.pdf >

PÖCHHACKER, Franz. (2008) "Interpreting as Mediation." En: Valero Garcés, Carmen \& Anne Martin (eds.) 2008. Crossing Borders in Community Interpreting: Definitions and dilemmas. Ámsterdam \& Filadelfia: John Benjamins, pp. 9-26.

PONTRANDOLFO, Gianluca. (en prensa) "Aproximación gradual a la traducción jurídica: un recorrido didáctico.” JoSTrans, Journal of Specialised Translation Issue 26/2016.

RodRíGUEZ CALA, Ana \& Núria Llevot Calvet. (2011) "La mediación intercultural: una realidad en los hospitales.” En: García Castaño, Francisco Javier \& Nina Kressova (eds.) 2011. Actas del I Congreso Internacional sobre Migraciones en Andalucía. Granada: Instituto de Migraciones, pp. 1065-1073.

SERIANNI, Luca. (2005) Un treno di sintomi: i medici e le parole: percorsi linguistici nel passato e nel presente. Milán: Garzanti.

SERIANNI, Luca. (2007) Italiani scritti. Bolonia: Il Mulino.

Straniero Sergio, Francesco. (en prensa) Double Renditions in Simultaneous Interpreters' Output. A Corpus-based Research. Trieste: EUT.

VALERO GARCÉs, Carmen. (2008) Formas de mediación intercultural. Traducción e Interpretación en los servicios públicos. Conceptos, datos, situaciones y práctica. $2^{a}$ edición. Granada: Comares.

VAlERO GARCÉS, Carmen; Bárbara Navaza \& Lissie Wahl-Kleiser. (2014) "Introspectiva y retrospectiva de la traducción e interpretación médica." Panace@ 15:40, pp. 165-167. Versión electrónica: <http://www.medtrad.org/ panacea/IndiceGeneral/n40-editorial.pdf >

ViagGio, Sergio. (1995) "The Praise of Sight Translation (and squeezing the Last Drop thereout of)." The Interpreters' Newsletter 6, pp. 33-42.

VIEZZI, Maurizio. (1989) "Sight Translation: An Axperimental Analysis." En: Dodds, John (ed.) 1989. Aspects of English: Miscellaneous Papers for English Teachers and Specialists. Udine: Campanotto, pp. 109-140.

Vivanco Cervero, Verónica. (2006) El español de la ciencia y la tecnología. Madrid: Arco/Libros.

WADENSJÖ, Cecilia. (1998) Interpreting as interaction. Londres \& Nueva York: Longman. 
WEBER, Wilhelm K. (1990) "The Importance of Sight Translation in an Interpreter Training Program.” En: Bowen, David \& Margareta Bowen (eds.) 1990. Interpreting - Yesterday, Today, and Tomorrow, Ámsterdam \& Filadelfia: John Benjamins, pp. 44-52.

ZORZI, Daniela. (2007) "Note sulla formazione dei mediatori linguistici." Studi di Glottodidattica 1:1, pp. 112-128.

\section{NOTA BIOGRÁFICA / BIONOTE}

GianluCa Pontrandolfo es doctor en ciencias de la interpretación y traducción y profesor asociado de traducción general y especializada españolitaliano en el Departamento IUSLIT (Departamento de Ciencias Jurídicas, del Lenguaje, de la Interpretación y de la Traducción) de la Universidad de Trieste. Combina su actividad profesional de traductor autónomo con la investigación académica, que se centra en los aspectos terminológicos y fraseológicos del lenguaje jurídico, enfocados desde una perspectiva contrastiva (español, italiano, inglés) y mediante la lingüística de corpus. Su tesis doctoral abarca el tema de la fraseología especializada en un corpus trilingüe de sentencias penales (COSPE), en línea con las demás publicaciones sobre lengua y derecho. Sus intereses de investigación se centran además en géneros especializados y análisis textual para la traducción, didáctica de la traducción, lingüística de corpus. Es miembro agregado del CERLIS, Centro de Investigación sobre las lenguas de especialidad, de la Universidad de Bérgamo.

Dr. GianluCA Pontrandolfo is currently Adjunct Professor at the University of Trieste (IUSLIT, Department of Legal Language, Interpreting and Translation Studies), where he lectures on general and specialised translation from Spanish into Italian. He combines his activity as free-lance translator with his academic and research projects. His research activity focuses on translation-oriented legal terminology and phraseology, from a contrastive (Spanish, Italian, English) and corpus perspective. In line with his $\mathrm{PhD}$ thesis, which deals with a quali-quantitative study of legal phraseology in a trilingual corpus of criminal judgments (COSPE), his publications focus on the relationship between language and law. His research interests also focus on specialized genres, including textual analysis applied to translation, translator training and corpus linguistics. He is member of the CERLIS (Research Centre on Languages for Specific Purposes) of the University of Bergamo (Italy). 